\title{
Hepatic Abnormalities in Patients with Chronic Granulomatous Disease
}

Nadeem Hussain, ${ }^{1,2,7} *$ Jordan J. Feld, ${ }^{1,7} *$ David E. Kleiner, ${ }^{5,7}$ Jay H. Hoofnagle, ${ }^{1,7}$ Reyes Garcia-Eulate, ${ }^{3,7}$ Sushil Ahlawat, ${ }^{2,7}$ Deloris E. Koziel, ${ }^{4,7}$ Victoria Anderson, ${ }^{6,7}$ Dianne Hilligoss, ${ }^{6,7}$ Peter Choyke, ${ }^{3,7}$ John I. Gallin, ${ }^{6,7}$ T. Jake Liang, ${ }^{1,7}$ Harry L. Malech,, 7 Steven M. Holland, ${ }^{6,7}$ and Theo Heller ${ }^{1,7}$

From the ${ }^{1}$ Liver Diseases Branch, National Institute of Diabetes c 'Digestive \& Kidney Diseases, Bethesda, MD; the ${ }^{2}$ Department of Gastroenterology, Georgetown University Hospital, Washington, DC; the ${ }^{3}$ Diagnostic Radiology Department and ${ }^{4}$ Center for Biostatistics, Warren Grant Magnuson Clinical Center, Bethesda, MD; the ${ }^{5}$ Laboratory of Pathology, National Cancer Institute, Bethesda, MD; the ${ }^{6}$ National Institute of Allergy and Infectious Diseases, Bethesda, MD; and the ${ }^{7}$ National Institutes of Health, Bethesda, MD.

*These authors contributed equally to this work.

Address reprint requests to: Theo Heller, Liver Diseases Branch/NIDDK/NIH, Building 10, Room 9B16, 10 Center Drive, MSC 1800, Bethesda, MD 208921800.

E-mail: theller@nih.gov; fax: 301-402-0491.

\begin{abstract}
Chronic granulomatous disease (CGD) is a rare congenital disorder characterized by repeated bacterial and fungal infections. Aside from a high incidence of liver abscess, little is known about hepatic involvement in CGD. The aim of this study was to describe the spectrum of liver abnormalities seen in CGD. The charts of 194 patients with CGD followed at the NIH were reviewed, with a focus on liver abnormalities. Liver enzyme elevations occurred on at least one occasion in $73 \%$ of patients during a mean of 8.9 years of follow-up. ALT elevations were generally transient. Although transient alkaline phosphatase (ALP) elevations were also common, persistent ALP elevations lasting up to 17.6 years were seen in $25 \%$ of patients. Liver abscess occurred in $35 \%$ of patients. Drug-induced hepatotoxicity was documented in $15 \%$ of patients but likely occurred more frequently. Hepatomegaly was found in 34\% and splenomegaly in $56 \%$ of patients. Liver histology showed granulomata in $75 \%$ and lobular hepatitis in $90 \%$ of specimens. Venopathy of the portal vein was common $(80 \%)$ and associated with splenomegaly. Venopathy of the central vein was also common (63\%) and was associated with the number of abscess episodes. Nodular regenerative hyperplasia (NRH) was seen in 9 patients, including 6 of 12 autopsy specimens. Conclusion: Liver enzyme abnormalities occur frequently in patients with CGD. In addition to liver abscesses and granulomata, drug hepatotoxicity is likely underappreciated. Vascular lesions such as venopathy and-to a lesser extent- $\mathrm{NRH}$ are common. The cause and clinical consequences of venopathy await prospective evaluation.
\end{abstract}




\section{INTRODUCTION}

Chronic granulomatous disease (CGD) is a rare inherited disorder occurring in 1 in 250,000 individuals. ${ }^{1}$ The underlying defect is a loss or inactivation of a component of the reduced NADPH complex, resulting in a defective oxygen metabolic burst with inadequate production of superoxide and peroxide and, perhaps most importantly, an inability of phagocytic cells to kill certain bacteria and fungi. ${ }^{2}$ These defects predispose affected individuals to recurrent infectious complications and significantly reduced long-term survival. ${ }^{3}$

Four principal CGD genotypes have been described, with important differences in phenotypic expression. Because the gp91 ${ }^{\text {phox }}$ genotype is X-linked and accounts for $70 \%$ of patients in large cohort studies, CGD has a significant male predominance. ${ }^{4}$ The other described genotypes, ${ }^{\mathrm{p} 22 \mathrm{phox}}, \mathrm{p} 47^{\text {phox }}$, and $\mathrm{p} 67^{\mathrm{phox}}$, are autosomal recessive. ${ }^{4}$ In addition to being more common, X-linked CGD (gp91 ${ }^{\text {phox }}$ ) portends a worse prognosis with a higher annual mortality compared with the other genotypes. ${ }^{1}$ The reduced NADPH is composed of cell membrane $\left(\mathrm{p} 22^{\text {phox }}\right.$, gp91 ${ }^{\text {phox }}$ ) or cytoplasmic moieties $\left(\mathrm{p} 47^{\text {phox }}, \mathrm{p} 67^{\text {phox }}\right){ }^{1}$

Although pulmonary infections predominate in CGD, with $79 \%$ of a 368-patient registry reporting a history of pneumonia, other sites are also commonly affected, including suppurative adenitis (53\%), subcutaneous abscess (42\%), liver abscess (27\%), and osteomyelitis $(25 \%) .^{5}$ Catalase-positive organisms are particularly problematic in CGD, because they are thought to prevent impaired CGD phagocytes from using microbial hydrogen peroxide. ${ }^{5}$ Staphylococcal species are the most common organisms seen, particularly in liver abscesses, where they account for over $50 \%$ of infections. ${ }^{6}$ Gram-negative rods (Serratia marcescens, Burkholderia cepacia) and fungi (Aspergillus species) are also commonly reported. ${ }^{6}$

Liver abscesses typically present with fever with or without abdominal pain and are often accompanied by constitutional symptoms. In patients with CGD, abscess recurrence usually results from new primary infection rather than relapse. ${ }^{6}$ Radiological

findings of abscess may differ in CGD, as described recently. ${ }^{7}$ Aggressive surgical management has been shown to be more effective than medical intervention alone for large abscesses, reducing the mortality from $27 \%$ to $6 \% .^{8,9}$

With earlier diagnosis, IFN- $\gamma$ and antimicrobial prophylaxis, and aggressive surgical and medical management, mortality for patients with CGD has plummeted in just a few decades with many patients now surviving well into adulthood. ${ }^{9,10}$ Aside from the association with liver abscess, little is known about liver disease in CGD. Because patients are living longer, the consequences of recurrent hepatic abscesses, complicated and prolonged courses of antimicrobials, and often-large hepatic resections may become more relevant. The goal of this retrospective study was to define the spectrum of liver disease and hepatopathology in a large cohort of well-characterized patients with CGD.

\section{PATIENTS AND METHODS}

A large cohort of patients with CGD has been followed at the Clinical Center of the $\mathrm{NIH}$ as part of a natural history protocol. All patients had the diagnosis of CGD 
confirmed by either nitroblue tetrazolium reduction or dihydrorhodamine oxidation. ${ }^{11}$ The specific gene defect was determined via immunoblotting, sequencing, or both whenever possible. All available patient charts as well as laboratory, pathological, microbiological, and radiological data were reviewed.

All radiological studies were reviewed by an abdominal radiologist with particular attention to liver contour and size, hepatic calcifications, abscesses, portal hypertensive changes, and spleen size. Hepatomegaly and splenomegaly were determined in relation to body size. A dedicated hepatopathologist reviewed all liver biopsy and wedge resection specimens. All histological specimens were examined at least $2 \mathrm{~cm}$ distant from liver abscesses to avoid reporting of peri-abscess changes.

Because data were collected longitudinally, liver enzyme elevations were categorized based on the predominant pattern over time. Enzyme elevations returning to baseline or normal within 3 months were deemed transient rises, while those persisting for longer than 3 months were defined as persistent. All enzyme elevations were recorded in relationship to normal values: normal, 1-2 times the upper limit of normal (ULN), 2-5 times ULN, 5-10 times ULN, and >10 times ULN. Because alkaline phosphatase (ALP) is elevated in childhood due to bone growth, for patients 14 years or younger, a ULN of ALP of $400 \mathrm{IU} / \mathrm{L}$ was used, and thereafter evaluations were based on the adult ULN value of $116 \mathrm{IU} / \mathrm{L}$. In the majority of cases, ALP elevations were confirmed to be of liver origin by a corresponding elevation of $\gamma$-glutamyltranspeptidase on at least one occasion. The following definitions were used to define liver enzyme patterns:

1. Persistent hepatocellular injury: ALT $\geq$ ULN for $>3$ months with ALP elevation of $\leq 1$ category of elevation below that of ALT (e.g., ALT 2-5X for 6 mo with ALP $\leq 1-2 X$ ULN).

2. Transient hepatocellular injury: ALT elevation $\geq 2$ category difference more than ALP elevation (e.g., ALT $>10 X$ ULN with ALP $\leq 2-5 X$ ULN) with spontaneous return to normal or previous baseline within 3 months.

3. Persistent cholestatic injury: ALP $\geq$ ULN for $>3$ months with $\leq 2$ flares of ALT to equal category of ALP elevation (e.g., ALP 2-5 X ULN for 6 mo with $\leq 2$ ALT flares to 2-5X ULN).

4. Transient cholestatic injury: ALP elevation $\geq 2$-category difference more than ALT elevation (e.g., ALP $>10 X$ ULN with ALT $\leq 2-5 X$ ULN) with spontaneous return to normal or previous baseline within 3 months.

5. Persistent cholestatic injury with ALT flares: ALP $\geq$ ULN for $>3$ months with ALT flares beyond those described in category 3 (e.g., ALP 2-5X ULN with 5 ALT flares to 5-10X ULN).

6. Mixed injury: ALP and ALT with < 2-category difference of magnitude of elevation (e.g., ALT 1-2X ULN with ALP 2-5X ULN)

7. No significant elevation: ALP and ALT persistently in normal range or $\leq$ 2 elevations to $\leq 1-2 \mathrm{X}$ ULN during follow-up.

Previous reports in the literature define only individual values rather than longitudinal data. To better appreciate the effects of prolonged enzyme elevations over time, the above definitions were developed.

Categorical variables were compared using the $\chi^{2}$ or Fisher exact test, and continuous variables were compared using the Student $t$ test or one-way ANOVA. A $P$ value of less 
than 0.05 was considered statistically significant. Univariate and multivariate logistic regression were per-formed using SAS software.

\section{RESULTS}

A total of 194 patients with CGD were evaluated. The majority of patients were male (n $=150[77 \%])$ and most were Caucasian $(\mathrm{n}=155$ [80\%]) (Table 1). Other ethnicities were also represented, including African American $(n=22[11 \%])$, Hispanic $(n=12$ $[6 \%])$, Asian $(n=5[2 \%])$. The mean age at last follow-up was 19.5 years (range 1.363.9), and patients were followed for a mean of 8.9 years. The CGD genotype distribution was as follows: gp $91^{\text {phox }} \mathrm{n}=114(59 \%), \mathrm{p} 47^{\text {phox }} \mathrm{n}=56(29 \%), \mathrm{p} 22^{\text {phox }} \mathrm{n}=$ $4(2 \%), \mathrm{p} 67^{\text {phox }}, \mathrm{n}=3(2 \%)$, and unknown, $\mathrm{n}=17(8 \%)$. Twelve patients $(6 \%)$ had previously undergone bone marrow transplantation as curative therapy for CGD. A total of 23 patients died during follow-up, all of infectious complications.

Liver enzyme abnormalities were common, occurring in $141(73 \%)$ patients on at least one occasion during follow-up. Various patterns of both ALT and ALP elevations were seen. The majority of ALT elevations were transient, returning to normal values within 3 months; however, 4 patients had persistent mild ALT elevations (1-2 X ULN) for up to 18.6 years. In contrast, 49 (25\%) patients had persistent elevations of ALP (mean 198 IU/ L), 13 of which were at least 2-5 X ULN (mean 233 IU/L). The persistent ALP elevations lasted for a mean of 5.1 years (range 0.8-17.6), accounting for an average of $48 \%$ (range $7 \%-100 \%$ ) of the duration of follow-up.

Transient ALT elevations occurred in 116 patients $(60 \%)$ ranging from mild $(<2 \mathrm{X}$ ULN) to moderate (2-5X ULN) in 38 and 35 patients, respectively, while 43 (22\%) patients had more significant ALT elevations (>5X ULN), including 25 (9\%) patients with at least one ALT value of >10X ULN. Patients had a mean of 2.8 (range 0-26) ALT elevations or 0.51 (range 0-6.2) ALT elevations per year of follow-up.

Transient elevations of ALP were also common, occurring in 97 (50\%) patients. Forty (21\%) patients had only mild ALP elevations (1-2X ULN), while 37 (19\%) had moderate (2-5X ULN) and $20(10 \%)$ had severe (>5X ULN) ALP elevations. Seven (4\%) patients had at least one ALP elevation to >10X ULN. On average, patients had 1.7 (range 0-23) elevations of ALP or 0.6 (range 0-25) elevations of ALP per year of follow-up.

The patterns of liver enzyme elevation were categorized as described in the methods section to take into account the fact that patients had multiple and often concomitant elevations of both transaminases and ALP. These categorizations provide a more general summary of the pattern of liver enzyme elevations over time. Using these definitions, $45(23 \%)$ patients had a predominantly cholestatic pattern of elevation, 7 (4\%) transient and $38(20 \%)$ persistent. Of those with a persistent cholestatic pattern, 14 (37\%) had significant ALT flares superimposed upon chronic ALP elevation. A predominantly hepatocellular pattern and a mixed pattern of elevation were each seen in 35 patients (18\%). Seventy-nine patients $(41 \%)$ had normal or near normal liver enzymes throughout follow-up. 


\section{Etiology}

A small number of patients had coexisting liver disease not directly related to CGD. Four patients had antibodies to $\mathrm{HCV}$, and 3 were positive for hepatitis $\mathrm{C}$ RNA. Two patients had chronic hepatitis B infection, 2 had nonalcoholic steatohepatitis on liver biopsy, 2 had chronic cholestasis secondary to total parenteral nutrition, and 2 had hepatic graft-versus-host disease as a com-plication of previous bone marrow transplantation. Although not directly related to CGD, it is notable that two of the HCVinfected patients likely acquired infection from contaminated blood products, and total parenteral nutrition as well as bone marrow transplant were used as treatment for complications of CGD.

Just over one third of patients $(n=69)$ had a history of liver abscess, and 16 patients had multiple episodes (range 0-4), resulting in a total of 90 abscess episodes reported in the entire cohort. Multiple concurrent abscesses were common but were counted as a single abscess episode for analysis. Patients with a history of liver abscess had significantly more ALT and ALP elevations than those with no history of abscess ( $\mathrm{P}<0.05$ ). A history of abscess was associated with ALP elevations $>5 \mathrm{X}$ ULN $(\mathrm{P}<0.05)$ and ALT elevations $>10 \mathrm{X}$ ULN $(\mathrm{P}<0.05)$. Microbiological data were available for 27 abscesses. Although Staphylococcus species were the most common organisms identified (17 Staphylococcus aureus, 11 coagulase-negative staphylococci, 7 both), Staphylococcus aureus was found as the unique organism in only 5 cases. Seventeen abscesses were polymicrobial. Six patients had fungal abscesses due to Candida, Aspergillus, and Cryptococcus species. Mycobacterium fortuitum was found in 1 case, as were Nocardia, Burkholdia cepacia, Enterobacter, Enterococcus, pediococcus, lactobacillus and Aerobacter species. Fifty-two of the 69 (75\%) patients underwent hepatic resection, while 8 were managed with percutaneous drainage and 9 underwent both procedures. Similar to previous reports, a high rate of surgical complications was encountered, occurring in 34 (49\%) patients. ${ }^{9,12}$

After liver abscess, drug-induced hepatotoxicity was the most commonly identified cause of liver enzyme elevation. Enzyme elevation was attributed to medication use if all other etiologies were excluded and improvement was documented with discontinuation of the suspected offending agent or if a typical pattern of hepatotoxicity was noted on liver biopsy. Based on these criteria, a suspicion of drug-induced liver disease was documented in the chart of 29 patients, and 2 patients had total parenteral nutrition-induced cholestasis. Antimicrobial agents were the most commonly implicated medications. Antifungals were the suspected cause in 9 patients (voriconazole 3, itraconazole 5, amphotericin 1), followed by oxacillin in 8 , sulfonamides in 4, cephalosporins in 4 and individual cases from cyclosporin A, tacrolimus, levofloxacin, chloramphenicol, and possibly IFN- $\gamma$. In numerous cases, more than one possible medication was suspected; however, all agents were stopped simultaneously, making definitive identification of the culprit agent difficult. In $93 \%$ (27/29) of cases, drug toxicity caused an ALT elevation: purely hepatocellular in 11 patients $(38 \%)$, a mixed pattern in 10 patients $(35 \%)$, and a persistent cholestatic pattern with ALT flares in 6 patients $(21 \%)$. 


\section{Other Abnormalities}

In addition to enzyme elevations, other hepatic abnormalities were noted on abdominal imaging, including ultrasound, CT, and magnetic resonance imaging. Of the 165 patients with radiological studies available for review, 56 (34\%) had hepatomegaly; 92 (56\%) had splenomegaly, including 5 with massive splenomegaly; and 46 (28\%) had enlargement of both the liver and spleen. Calcifications were seen in the liver in 41 $(25 \%)$ patients and in the spleen in $6(4 \%)$ patients. Other findings included retroperitoneal adenopathy in $41(25 \%)$ patients and splenic abscess in $3(2 \%)$ patients. Patients with a previous history of liver abscess were more likely to develop hepatomegaly and/or splenomegaly $(\mathrm{P}<0.05)$ and were more likely to have hepatic calcifications on imaging $(\mathrm{P}<0.0001)$. Patients with hepatomegaly and/or splenomegaly most commonly had a cholestatic pattern of liver enzyme elevation.

\section{Associations}

To evaluate the predictors of the development of splenic and hepatic enlargement, univariate and multivariate logistic regression were performed (Table 2). By univariate analysis, male sex, genotype gp91 ${ }^{\text {phox }}$, an increasing number of episodes of liver abscess, higher mean ALT as well as increasing number of ALT and ALP elevations were predictive of the development of splenomegaly, while a pattern of normal or nearnormal enzymes during follow-up was associated with a decreased risk. Multivariate logistic regression analysis revealed that genotype gp91 ${ }^{\text {phox }}$ (OR 6.38, 95\% CI 3.07 11.9; $\mathrm{P}<0.0001$ ), an increasing number of liver abscesses (OR 1.93, 95\% CI 1.12-3.32; $\mathrm{P}=0.019)$, and total ALT elevations (OR 1.13, 95\% CI 1.01-1.26; $\mathrm{P}=0.027)$ remained predictive, and normal or near-normal enzymes remained protective $(\mathrm{P}=0.048)$ for the development of splenomegaly. CGD genotype appeared to play an important role, because $71(62 \%)$ patients with gp91 ${ }^{\text {phox }}$ had splenic enlargement, compared with only $15(26 \%)$ patients with the $\mathrm{p} 47^{\text {phox }}$ mutation $(\mathrm{P}<0.01)$.

In contrast, by univariate analysis, black race, a history of drug hepatotoxicity, more episodes of liver abscess, total ALT and ALP elevations, and a cholestatic pattern of liver injury were associated with hepatomegaly. Multivariate analysis revealed that only black race (OR 7.04, 95\% CI 2.47-20.1; $\mathrm{P}=0.03$ ) and increasing number of ALT elevations (OR 1.21, 95\% CI 1.06-1.38; P < 0.0001) were associated with liver enlargement (Table 2).

Certain factors were predictive of the pattern of liver enzyme elevation seen over time (Table 3). A cholestatic pattern of injury was associated with increasing episodes of liver abscess (OR 1.59, 95\% CI 1.03-2.50; $\mathrm{P}=0.038$ ) and with CGD genotype. In patients with the gp91 $1^{\text {phox }}$ mutation, a cholestatic pattern was commonly seen $(30 \%)$ (OR 3.10, 95\% CI 1.43-6.72; $\mathrm{P}=0.004)$, whereas in those with the $\mathrm{p} 47^{\mathrm{phox}}$ mutation, this pattern was seen in only 5 patients $(9 \%)(\mathrm{OR} 0.23,95 \%$ CI $0.085-0.63$; P $=0.004)$ $(\mathrm{P}<0.01)$ (Table 1). Patients with the $\mathrm{p} 47^{\text {phox }}$ mutation most commonly had a mixed pattern of enzyme elevation. However, increasing patient age was the only factor associated with a mixed pattern (OR $1.11,95 \%$ CI $1.06-1.16 ; \mathrm{P}=0.01)$ by multivariate analysis (Table 3 ). Only a history of drug toxicity was independently associated with a hepatocellular pattern (OR 3.59, 95\% CI 1.51-8.54; $P=0.004)$. Normal or near normal enzymes were seen most commonly in younger patients, those with a lower body mass 
index and in patients with a genotype other than $\mathrm{p} 47^{\text {phox }}$ or gp91 ${ }^{\text {phox }}$. Not surprisingly, this pattern was much less common in patients with a history of liver abscess, drug hepatotoxocity, hepatomegaly and/or splenomegaly (data not shown). The factors associated with a history of liver abscess were increased age, total ALP elevations (OR $1.29,95 \%$ CI 1.13-1.48; $P=0.0002$ ), total ALT elevations (OR 1.10, 95\% CI 1.02-1.18; $P=0.011$ ), and a mixed enzyme pattern (OR 2.38, 95\% CI 1.12-5.05; $P=0.024$ ). However, multivariate analysis revealed that only age (OR $1.2995 \%$ CI 1.08-1.56, $P=$ 0.0006 ) and the number of ALP elevations (OR $1.0495 \%$ CI 1.00-1.07, $P=0.04$ ) remained significant (Table 2). CGD genotype was not associated with a history of liver abscess.

\section{Histology}

A total of 88 liver specimens from 38 patients were available for histological evaluation. Liver tissue was reviewed after hepatic wedge resection in 59 cases, from autopsy specimens in 12 cases, and from diagnostic core needle liver biopsies in 17 cases. Multiple specimens were reviewed from 16 patients (range 2-8 per patient), largely due to the need for multiple hepatic resections at the time of liver abscess. To ensure that findings were reflective of true liver pathology rather than local periabscess phenomena, only tissue from $\geq 2 \mathrm{~cm}$ away from the abscess capsule was evaluated. In 19 specimens, there was inadequate uninvolved liver tissue for review.

A variety of pathological changes were identified in the needle and wedge biopsies (Table 4). Some tissue specimens could not be evaluated for all pathological findings and consequently the denominator used for comparisons reflects the number of specimens that had adequate tissue and staining to evaluate the specific finding in question. Portal and/or lobular chronic hepatitis was seen in almost all evaluable specimens $(95 \%)$. Non-necrotizing granulomata and evidence of liver abscess were also common (75\% and $73 \%$, respectively) (Fig. 1).

Inflammatory duct lesions similar to those seen in primary sclerosing cholangitis were seen in 20\% (14/70) of samples in 10 different patients. These consisted mainly of narrowing of large ducts accompanied by concentric fibrosis, periductular edema, and lymphocytic inflammation. Rare obliterative scars and copper accumulation were present, but extensive ductopenia was not seen (see Fig. 2). Evidence of acute cholangitis (neutrophils within interlobular bile ducts) was seen in 11/70 (16\%) specimens in 9 different patients and was associated with the total number of episodes of liver abscess (OR 3.08, 95\% CI 1.13-8.40; $P=0.028$ ).

Abnormalities of both the portal and central veins were common (Fig. 1). A portal venopathy consisting of narrowing or complete obliteration of portal veins on MassonTrichrome staining was present in 57/71 (80\%) specimens from 24 patients. In some cases, this was associated with hypertrophy of the vein wall. Portal venopathy was consistent over time in the 10 patients for whom more than one sample was available for review. Similar changes were found in the central veins in 44/70 (63\%) samples from 20 patients and again were seen repeatedly in serial samples from individual patients. Portal and central venopathy were correlated $(P=0.02)$, and both were present in $41 / 70(59 \%)$ biopsy samples from 19 patients. Total episodes of liver abscess and the number of ALP elevations were associated with central venopathy. However, by 
multivariate analysis only the total episodes of liver abscess was significant (OR 9.35, 95\% CI 1.04-83.3; $P=0.046$ ) (Table 5). Splenomegaly was the only independent predictor of portal venopathy (OR 14.67, 95\% CI 1.83-117.7; $P=0.016$ ).

Nodular regenerative hyperplasia (NRH) was seen in 4 patients, one of whom later died and was confirmed to have NRH on autopsy. Of these, 1 of 4 patients had portal venopathy, and 2 had both central and portal vein changes. Reticulin staining was performed for only 30 specimens from 25 patients. Early changes of NRH may be missed on small biopsy samples, particularly without reticulin staining. Total ALP elevations were associated with NRH on biopsy or at autopsy (OR 1.20, 95\% CI 1.00$1.45 ; P=0.045$ ) (Table 5).

Cirrhosis was not found in any patients. Bridging fibrosis was noted in 2 patients, 1 with hepatitis $\mathrm{C}$ infection and 1 with nonalcoholic steatohepatitis.

Other abnormalities noted in individual biopsy specimens included sepsis-related cholestasis, drug-induced liver injury, hepatic infarction, aspergillosis, and cat-scratch disease. Sinusoidal dilatation was seen in $22(38.5 \%)$ samples.

Among the 12 autopsy specimens, NRH was seen in $6(50 \%)$ cases, and associated venopathy was found in 5 of these: 1 portal, 2 central, 2 portal and central (Fig. 1). In autopsy cases without NRH, portal venopathy was seen in 1, and both central and portal changes were seen in 3 specimens. Two of the autopsy specimens had no significant hepatic pathology.

\section{DISCUSSION}

With the marked improvement in prophylaxis and management of infections, patients with CGD are now typically living into adulthood. Although liver abscesses and granulomatous hepatitis have been described, little else is known about hepatic involvement in CGD. ${ }^{13}$ A large cohort of well-characterized patients with CGD was reviewed to better describe the clinical, laboratory, and histological manifestations of CGD in the liver.

Liver enzyme abnormalities were very common, occurring on at least one occasion in $73 \%$ of patients. Both transient and persistent elevations of ALP occurred, but elevations of aminotransferases were predominantly transient. The elevations seen were significant, reaching at least 5X ULN for ALP in 13\% of patients and for ALT in 22\% of patients. A cholestatic pattern of injury was slightly more common than hepatocellular or mixed patterns ( $23 \%$ vs. $18 \%$ vs. $18 \%$, respectively; P value not significant), and $41 \%$ of patients had only mild or no elevations.

Although liver abscess was the most common cause of liver enzyme elevation, this was the etiology in fewer than half $(48.5 \%)$ of the patients. Drug hepatotoxicity was documented in 29 cases but likely occurred much more frequently. Enzyme abnormalities were attributed to drug-induced liver injury only when all other etiologies were reasonably excluded, when resolution occurred with medication withdrawal, when a diagnosis of suspected drug hepatotoxicity was noted in the chart, or when a pattern 
consistent with drug-induced liver injury was seen on a liver biopsy specimen. Most patients with CGD remain on long-term antimicrobial prophylaxis and may receive other courses of therapy for active infections. ${ }^{10}$ It is likely that many of the enzyme elevations seen were attributable to one or more medications; however, this could not be ascertained with certainty. It is often difficult to determine a precise cause for liver enzyme elevations, particularly in the setting of systemic infection. ${ }^{14}$ Mild enzyme elevations from suspected drug hepatotoxicity do not always prompt discontinuation of potentially life-saving therapy. Although this is certainly reasonable in the acute setting, it is unknown whether continued therapy (for years in some cases) may lead to progressive liver disease. Several patients had significant abnormalities on liver biopsy, including portal and central venopathy as well as $\mathrm{NRH}$, all of which have been associated with drug-induced liver injury in previous reports ${ }^{14,15}$ but not described previously in CGD. ${ }^{13}$ Although such changes may be the consequence of repeated hepatic and systemic infections, clarifying the role of chronic drug hepatotoxicity will have important implications for the management of CGD and other chronic diseases.

In addition to liver enzyme elevations, many patients had hepatic and/or splenic enlargement. Although hepatosplenomegaly could result from causes other than chronic liver disease, it is notable that both lesions were associated with more frequent episodes of liver abscess and liver enzyme elevations. In addition, the patients with normal or near-normal liver tests throughout follow-up were unlikely to develop either hepatomegaly or splenomegaly. Liver enlargement was associated with a cholestatic pattern of liver injury. Although ALP elevation is typically associated with cholestasis and biliary abnormalities, it may also be seen with hepatic infiltration or distortion of hepatic architecture. ${ }^{16}$ Hepatomegaly and ALP elevation are common in the setting of hepatic infection, particularly by fungal or mycobacterial organisms. ${ }^{16,17}$ Repeated liver abscesses as well as hepatic resections may significantly alter hepatic architecture, potentially resulting in ALP elevation, hepatomegaly and/or splenomegaly, and histological changes.

In addition to the previously described granulomata, abscesses, and portal and lobular hepatitis, some novel pathological lesions were seen. ${ }^{13}$ Significant abnormalities of the portal or central veins were found in over $80 \%$ of the biopsy specimens examined. In addition, NRH was noted in half of the autopsies and over $23 \%$ of the biopsy specimens evaluated. Although the pathogenesis is incompletely understood, $\mathrm{NRH}$ is a cause of noncirrhotic portal hypertension postulated to result from a primary vasculopathy and obliteration of small portal branches. ${ }^{18-20}$ The diagnosis is frequently overlooked due to slow evolution, lack of impairment of hepatic synthetic function, and the need for special staining. ${ }^{21}$ Increased resistance to blood flow within the hepatic sinusoids results in portal hypertensive changes. ${ }^{22,23}$ It is possible that the vascular changes identified in a high proportion of biopsies may progress, ultimately leading to the development of NRH.

The cause of the vascular abnormalities is unclear. Recurrent liver abscesses and resections may alter the hepatic architecture and lead to changes in both liver inflow and outflow with potential consequences to small portal and hepatic venules over time. Alternatively, granulomata may alter hepatic architecture and lead to $\mathrm{NRH}$, a phenomenon that has been postulated to occur in primary biliary cirrhosis. ${ }^{24,25}$ Notably, central venopathy was associated with the number of episodes of liver abscess and portal venopathy was independently associated with splenomegaly. Medications have 
also been described to cause both vasculopathy and NRH. Consequently, the etiology may be multifactorial. ${ }^{26}$

The main limitation of this study is its retrospective nature. This limits findings to associative rather than causative. In addition, the cohort may reflect patients with more severe CGD as all included patients were referred to the NIH for evaluation.

In conclusion, liver enzyme elevations are common in patients with CGD, associated primarily with liver abscesses and drug hepatotoxicity. Over time, many patients develop hepatomegaly and/or splenomegaly, which correlate with episodes of liver abscess and frequency of liver enzyme elevation. Hepatic pathology includes granulomata and abscesses, but vascular abnormalities including $\mathrm{NRH}$ are also common. Whether vasculopathy results from repeated liver abscesses, drug hepatotoxicity, or other processes is currently unknown. As patients with CGD continue to live longer, understanding the consequences of hepatic pathology will become more important. In addition, CGD may serve as a model to evaluate the progression from vasculopathy to NRH.

\section{REFERENCES}

1. Jurkowska M, Bernatowska E, Bal J. Genetic and biochemical background of chronic granulomatous disease. Arch Immunol Ther Exp (Warsz) 2004; 52:113120.

2. Heyworth PG, Cross AR, Curnutte JT. Chronic granulomatous disease. Curr Opin Immunol 2003;15:578-584.

3. Johnston RB Jr. Clinical aspects of chronic granulomatous disease. Curr Opin Hematol 2001;8:17-22.

4. Ishibashi F, Nunoi H, Endo F, Matsuda I, Kanegasaki S. Statistical and mutational analysis of chronic granulomatous disease in Japan with special reference to gp91-phox and p22-phox deficiency. Hum Genet 2000;106: 473-481.

5. Winkelstein JA, Marino MC, Johnston RB Jr, et al. Chronic granulomatous disease. Report on a national registry of 368 patients. Medicine (Baltimore) 2000;79:155-169.

6. Guide SV, Stock F, Gill VJ, Anderson VL, Malech HL, Gallin JI, et al. Reinfection, rather than persistent infection, in patients with chronic granulomatous disease. J Infect Dis 2003;187:845-853.

7. Garcia-Eulate R, Hussain N, Heller T, Kleiner D, Malech H, Holland S, et al. CT and MRI of hepatic abscess in patients with chronic granulomatous disease. AJRAm J Roentgenol 2006;187:482-490.

8. Chen LE, Minkes RK, Shackelford PG, Strasberg SM, Kuo EY, Langer JC. Cut it out: managing hepatic abscesses in patients with chronic granulomatous disease. J Pediatr Surg 2003;38:709-713.

9. Lublin M, Bartlett DL, Danforth DN, Kauffman H, Gallin JI, Malech HL, et al. Hepatic abscess in patients with chronic granulomatous disease. Ann Surg 2002;235:383-391.

10. Marciano BE, Wesley R, De Carlo ES, Anderson VL, Barnhart LA, Darnell D, et al. Long-term interferon-gamma therapy for patients with chronic granulomatous disease. Clin Infect Dis 2004;39:692-699. 
11. Vowells SJ, Sekhsaria S, Malech HL, Shalit M, Fleisher TA. Flow cytometric analysis of the granulocyte respiratory burst: a comparison study of fluorescent probes. J Immunol Methods 1995;178:89-97.

12. Mulholland MW, Delaney JP, Simmons RL. Gastrointestinal complications of chronic granulomatous disease: surgical implications. Surgery 1983;94:569-575.

13. Nakhleh RE, Glock M, Snover DC. Hepatic pathology of chronic granulomatous disease of childhood. Arch Pathol Lab Med 1992;116:71-75.

14. Zimmerman HJ. Drug-induced liver disease. Clin Liver Dis 2000;4:73 96, vi.

15. Al-Mukhaizeem KA, Rosenberg A, Sherker AH. Nodular regenerative hyperplasia of the liver: an under-recognized cause of portal hypertension in hematological disorders. Am J Hematol 2004;75:225-230.

16. Maldonado O, Demasi R, Maldonado Y, Taylor M, Troncale F, Vender R. Extremely high levels of alkaline phosphatase in hospitalized patients. J Clin Gastroenterol 1998;27:342-345.

17. Essop AR, Posen JA, Hodkinson JH, Segal I. Tuberculosis hepatitis: a clinical review of 96 cases. Q J Med 1984;53:465-477.

18. Reshamwala PA, Kleiner DE, Heller T. Nodular regenerative hyperplasia: not all nodules are created equal. HEPATOLOGY 2006;44:7-14.

19. Kondo F, Koshima Y, Ebara M. Nodular lesions associated with abnormal liver circulation. Intervirology 2004;47:277-287.

20. Shimamatsu K, Wanless IR. Role of ischemia in causing apoptosis, atrophy, and nodular hyperplasia in human liver. HEPATOLOGY 1997;26:343 350.

21. Ibarrola C, Colina F. Clinicopathological features of nine cases of noncirrhotic portal hypertension: current definitions and criteria are inadequate. Histopathology 2003;42:251-264.

22. Austin A, Campbell E, Lane P, Elias E. Nodular regenerative hyperplasia of the liver and coeliac disease: potential role of $\operatorname{IgA}$ anticardiolipin antibody. Gut 2004;53:1032-1034.

23. Young ID, Segura J, Ford PM, Ford SE. The pathogenesis of nodular regenerative hyperplasia of the liver associated with rheumatoid vasculitis. J Clin Gastroenterol 1992;14:127-131.

24. Matsumoto T, Kobayashi S, Shimizu H, Nakajima M, Watanabe S, Kitami N, et al. The liver in collagen diseases: pathologic study of 160 cases with particular reference to hepatic arteritis, primary biliary cirrhosis, autoimmune hepatitis and nodular regenerative hyperplasia of the liver. Liver 2000;20:366-373.

25. Colina F, Pinedo F, Solis JA, Moreno D, Nevado M. Nodular regenerative hyperplasia of the liver in early histological stages of primary biliary cirrhosis. Gastroenterology 1992;102:1319-1324.

26. Dubinsky MC, Vasiliauskas EA, Singh H, Singh H, Abreu MT, Papadakis KA, et al. 6-thioguanine can cause serious liver injury in inflammatory bowel disease patients. Gastroenterology 2003;125:298-303.

Abbreviations: ALP, alkaline phosphatase; CGD, chronic granulomatous disease; $\mathrm{NRH}$, nodular regenerative hyperplasia; ULN, upper limit of normal.

Supported by the Intramural Research Programs of the NIDDK, National Institute of Allergy and Infectious Diseases, Warren Grant Magnuson Clinical Center, National Cancer Institute, and NIH. 


\begin{tabular}{|c|c|c|c|c|c|c|}
\hline & Total & Ep91ptom & sp47man: & gp22, & sp67m & $\begin{array}{l}\text { Unknown } \\
\text { Genotype }\end{array}$ \\
\hline Number $(\mathrm{n})$ & 194 & 114 & 56 & 4 & 3 & 17 \\
\hline Age $(y r)$ & $19.5 \pm 10.8$ & $17.7 \pm 8.6^{*}$ & $23.9 \pm 12.4^{*}, \dagger$ & $26.2 \pm 13.3$ & $26.0 \pm 18.8$ & $13.9 \pm 10.8^{t}$ \\
\hline \multirow[t]{2}{*}{$\operatorname{Sex}(M / F)$} & $150 / 44$ & $106 / 8^{*}$ & $25 / 31^{*}, t$ & $3 / 1$ & $2 / 1$ & $14 / 3^{t}$ \\
\hline & $(77.3 / 22.7)$ & $(93.0 / 7.0)$ & $(44.6 / 55 / 4)$ & $(75 / 25)$ & $(66.6 / 33.3)$ & $(82.4 / 17.6)$ \\
\hline $\begin{array}{l}\text { Body mass index } \\
\text { Ethnicity }\end{array}$ & $20.9 \pm 5.9$ & $20.2 \pm 6.0$ & $23.0 \pm 5.5^{*}$ & $21.6 \pm 1.3$ & $22.4 \pm 10.9$ & $16.8 \pm 2.3^{*}$ \\
\hline Caucasian & $155(80.0)$ & $94(82.5)$ & $43(76.8)$ & $3(75)$ & $2(66.6)$ & $13(76.5)$ \\
\hline African American & $22(11.3)$ & $12(10.5)$ & $10(17.9)$ & 0 & 0 & 0 \\
\hline Hispanic & $12(6.2)$ & $5(4.4)$ & $3(5.5)$ & $1(25)$ & $1(33.3)$ & $2(11.8)$ \\
\hline Asian & $5(2.6)$ & $3(2.6)$ & 0 & 0 & 0 & $2(11.8)$ \\
\hline ALT (mean IU) $)^{5}$ & $32.9 \pm 35.6$ & $31.6 \pm 27.6$ & $37.9 \pm 51.5$ & $40.7 \pm 18.8$ & $43.7 \pm 45.3$ & $21.1 \pm 8.2$ \\
\hline AST (mean U) & $35.5 \pm 35.4$ & $33.9 \pm 20.8$ & $41.3 \pm 58.2$ & $34.7 \pm 9.7$ & $32.9 \pm 20.0$ & $28.9 \pm 10.8$ \\
\hline ALP (mean IU) & $191.3 \pm 132$ & $182.2 \pm 89.5$ & $208.9 \pm 200$ & $132.6 \pm 24.8$ & $312.4 \pm 200$ & $188.5 \pm 63.3$ \\
\hline Bilitubin (mean mg/dl) & $0.56 \pm 0.86$ & $0.45 \pm 0.23$ & $0.61 \pm 1.12$ & $0.48 \pm 0.20$ & $0.78 \pm 0.86$ & $0.37 \pm 0.15$ \\
\hline Total number of ALP elevations & $1.7 \pm 3.2$ & $1.7 \pm 2.8^{*}$ & $1.5 \pm 2.51$ & $9.5 \pm 11.5^{*}, \dagger$ & $3.6 \pm 4.7$ & $0.45 \pm 0.5$ \\
\hline $1-2 \times$ & $0.93 \pm 2.3$ & $0.75 \pm 1.5$ & $0.82 \pm 1.6$ & $8.75 \pm 10.8$ & $3.0 \pm 4.4$ & $0.35 \pm 0.5$ \\
\hline $2.5 \times$ & $0.57 \pm 1.6$ & $0.66 \pm 1.8$ & $0.54 \pm 1.4$ & $0.5 \pm 0.6$ & $0.33 \pm 0.6$ & $0.12 \pm 0.3$ \\
\hline $5-10 \times$ & $0.15 \pm 0.7$ & $0.20 \pm 0.8$ & $0.13 \pm 0.7$ & 0 & 0 & 0 \\
\hline$>10 x$ & $0.06 \pm 0.4$ & $0.08 \pm 0.5$ & $0.02 \pm 0.1$ & $0.25 \pm 0.5$ & $0.33 \pm 0.6$ & 0 \\
\hline Persistent ALP elevation & $49(25.3)$ & $38(33.3)^{*}$ & $10(17.9)^{*}$ & $1(25)$ & 0 & 0 \\
\hline Total number of ALT elevationsl & $2.8 \pm 4.3$ & $2.9 \pm 4.41$ & $2.8 \pm 4.0^{*}$ & $11 \pm 7.7^{*} . \dagger$ & $3.3 \pm 2.5$ & $0.29 \pm 0.7$ \\
\hline $1-2 \times$ & $1.64 \pm 2.5$ & $1.63 \pm 2.2$ & $1.73 \pm 2.8$ & $6.75 \pm 5.6$ & $1.0 \pm 1.0$ & $0.29 \pm 0.7$ \\
\hline $2-5 \times$ & $0.73 \pm 1.5$ & $0.75 \pm 1.5$ & $0.75 \pm 1.5$ & $3.0 \pm 3.6$ & $0.33 \pm 0.6$ & 0 \\
\hline $5-10 x$ & $0.30 \pm 0.8$ & $0.35 \pm 0.9$ & $0.20 \pm 0.4$ & $0.75 \pm 0.5$ & $1.67 \pm 2.9$ & 0 \\
\hline$>10 x$ & $0.15 \pm 0.4$ & $0.15 \pm 0.4$ & $0.16 \pm 0.4$ & $0.5 \pm 0.6$ & $0.33 \pm 0.6$ & 0 \\
\hline Persistent ALI elevation & $4(2.1)$ & 0 & $3(5.4)$ & 0 & $1(33.3)$ & 0 \\
\hline History of abscess & $69(34)$ & $39(34.2)$ & $19(33.9)$ & $3(75)$ & $3(100)$ & $5(29.4)$ \\
\hline Abscess episodes & $0.47 \pm 0.7$ & $0.44 \pm 0.7$ & $0.46 \pm 0.8$ & $1.3 \pm 1.0$ & $1.3 \pm 0.6$ & $0.3 \pm 0.5$ \\
\hline Hepatotoxicily & $29(14.9)$ & $15(13.2)$ & $11(19.6)$ & $2(50)$ & $1(33.3)$ & 0 \\
\hline Hepatomegaly & $56(34)$ & $38(33.3)$ & $14(25)$ & $2(50)$ & 0 & $2(11.7)$ \\
\hline Splenomegaly & $92(56)$ & $71(62.2)^{*}$ & $15(26.8)^{*}$ & $2(50)$ & $2(66.6)$ & $2(11.7)$ \\
\hline \multicolumn{7}{|l|}{ Enzyme pattern } \\
\hline Cholestatic persistent & $24(12.4)$ & $21(18.4)^{*}$ & $2(3,6)^{*}$ & $1(25)$ & 0 & 0 \\
\hline Cholestatic acute & $7(3.8)$ & $3(2.6)$ & 0 & 0 & 0 & $4(23.5)$ \\
\hline Cholestatic + flares & $14(7.3)$ & $11(9.6)$ & $3(5.4)$ & 0 & 0 & 0 \\
\hline Hepatocellular & $35(18)$ & $21(18.4)$ & $12(21.4)$ & $1(25)$ & $1(33.3)$ & 0 \\
\hline Mixed & $35(18)$ & $16(14.0)^{*}$ & $16(28.6)^{*}$ & $2(50)$ & $1(33.3)$ & 0 \\
\hline Nornal/Near-normal & $79(40.7)$ & $42(36.8)$ & $23(41.1)$ & 0 & $1(33.3)$ & $13(76.5)$ \\
\hline
\end{tabular}


Table 2. Univariate and Multivariate Logistic Regression Demonstrating Predictors of Hepatomegaly, Splenomegaly, and Liver Abscess

\begin{tabular}{|c|c|c|c|}
\hline Factor & OR* $(95 \% \mathrm{CI})$ & $\begin{array}{c}\text { Univariate } P \\
\text { Value }\end{array}$ & $\begin{array}{c}\text { Multivariate } \\
P \text { Value }\end{array}$ \\
\hline \multicolumn{4}{|l|}{ Splenomegaly } \\
\hline Genotype gp91 & $6.38(3.07-11.9)$ & $<0.0001$ & $<0.0001$ \\
\hline No. of abscess episodes & $1.93(1.12-3.32)$ & 0.0045 & 0.019 \\
\hline No. of ALT elevations & $1.13(1.01-1.26)$ & 0.0005 & 0.027 \\
\hline Normal enzyme pattern & $0.29(0.16-0.54)$ & $<0.0001$ & 0.049 \\
\hline Female versus male sex & $0.32(0.18-0.78)$ & 0.0081 & NS \\
\hline History of abscess & $2.13(1.17-3.87)$ & 0.0136 & NS \\
\hline Mean ALT & $1.02(1.01-1.04)$ & 0.0094 & NS \\
\hline Genotype gp47 & $0.47(0.22-0.99)$ & 0.0004 & NS \\
\hline No. of ALP elevations & $1.18(1.05-1.33)$ & 0.0068 & NS \\
\hline Persistent ALP elevation & $2.37(1.22-4.63)$ & 0.011 & NS \\
\hline \multicolumn{4}{|l|}{ Hepatomegaly } \\
\hline Black vs. Caucasian & $7.04(2.47-20.1)$ & 0.005 & 0.03 \\
\hline No. of ALT elevations & $1.21(1.06-1.38)$ & $<0.0001$ & $<0.0001$ \\
\hline Drug hepatotoxicity & $3.24(1.44-7.28)$ & 0.0044 & NS \\
\hline No. of abscess episodes & $1.83(1.20-2.72)$ & 0.0048 & NS \\
\hline No. of ALP elevations & $1.28(1.13-1.45)$ & 0.0001 & NS \\
\hline Persistent ALP elevation & $2.66(1.35-5.26)$ & 0.0049 & NS \\
\hline Cholestatic pattern & $2.21(1.10-4.45)$ & 0.026 & NS \\
\hline \multicolumn{4}{|l|}{ Liver abscess } \\
\hline Age $(\mathrm{yr})$ & $1.05(1.02-1.09)$ & 0.0005 & 0.029 \\
\hline No. of ALP elevations & $1.29(1.13-1.48)$ & 0.0002 & 0.003 \\
\hline No. of ALT elevations & $1.10(1.02-1.18)$ & 0.011 & NS \\
\hline Mixed enzyme pattern & $2.38(1.12-5.05)$ & 0.024 & NS \\
\hline
\end{tabular}




\begin{tabular}{|c|c|c|c|}
\hline Factor & OR* $(95 \% \mathrm{CI})$ & $\begin{array}{c}\text { Univariate } \\
P \text { Value }\end{array}$ & $\begin{array}{c}\text { Multivariate } \\
P \text { Value }\end{array}$ \\
\hline \multicolumn{4}{|l|}{ Cholestatic** } \\
\hline No. of abscess episodes & $1.59(1.03-2.50)$ & 0.04 & 0.038 \\
\hline Hepatomegaly & $2.21(1.10-4.45)$ & 0.026 & NS \\
\hline Genotype gp91 & $3.10(1.43-6.72)$ & 0.004 & NS \\
\hline Genotype gp47 & $0.23(0.05-0.63)$ & 0.005 & 0.004 \\
\hline \multicolumn{4}{|l|}{ Hepatocellular } \\
\hline Drug hepatotoxicity & $3.59(1.51-8.54)$ & 0.004 & 0.004 \\
\hline \multicolumn{4}{|l|}{ Mixed } \\
\hline Age & $1.11(1.07-1.16)$ & $<0.0001$ & $<0.0001$ \\
\hline Drug hepatotoxicity & $3.09(1.28-7.45)$ & 0.012 & NS \\
\hline History of abscess & $2.40(1.14-5.10)$ & 0.022 & NS \\
\hline No. of abscess episodes & $1.66(1.07-2.57)$ & 0.025 & NS \\
\hline Genotype gp47 & $2.29(1.07-4.92)$ & 0.033 & NS \\
\hline \multicolumn{4}{|l|}{ Normal/Near-normal } \\
\hline Age & $0.92(0.89-0.96)$ & $<0.0001$ & 0.009 \\
\hline Duration of follow-up & $0.94(0.90-0.99)$ & $<0.0001$ & $<0.0001$ \\
\hline Body mass index & $0.89(0.82-0.96)$ & 0.003 & NS \\
\hline Genotype gp91 & $0.23(0.08-0.71)$ & 0.008 & NS \\
\hline Genotype gp47 & $0.25(0.08-0.89)$ & 0.008 & NS \\
\hline
\end{tabular}




\begin{tabular}{|c|c|c|}
\hline \multicolumn{3}{|c|}{ Table 4. Histological Findings in the Liver } \\
\hline & Pathology Specimens & Number of Patients \\
\hline Number & 88 & 38 \\
\hline Specimens/patient & NA & $2.3(0-8)$ \\
\hline \multicolumn{3}{|l|}{ Specimen type } \\
\hline Needle & $17(19.3)^{*}$ & 15 \\
\hline Wedge & $59(67.0)$ & 22 \\
\hline Autopsy & $12(13.6)$ & 12 \\
\hline Lobular/Portal hepatitis & $67 / 71(94.4)$ & $29 / 31(93.5)$ \\
\hline Abscess & $55 / 76(72.4)$ & $23 / 30(76.7)$ \\
\hline Granulomata & $56 / 75(74.7)$ & $23 / 31(74.2)$ \\
\hline Duct lesions & $14 / 70(20.0)$ & $10 / 31(32.3)$ \\
\hline Acute cholangitis & $11 / 70(15.7)$ & $9 / 29(31.0)$ \\
\hline Vasculopathy & $60 / 71(84.5)$ & $25 / 31(80.6)$ \\
\hline Central & $44 / 70(62.9)$ & $20 / 30(66.7)$ \\
\hline Portal & $57 / 71(80.3)$ & $24 / 31(77.4)$ \\
\hline Both & $41 / 70(57.1)$ & $19 / 30(63.3)$ \\
\hline $\mathrm{NRH}$ & $10 / 42(23.8) \dagger$ & $9 / 29(31.0)$ \\
\hline Cirrhosis/Bridging fibrosis & $2 / 27(7.4) \ddagger$ & $2 / 19(10.5)$ \\
\hline \multicolumn{3}{|c|}{$\begin{array}{l}\text { *Percentage of those specimens with each specific finding as a proportion of those } \\
\text { with adequate tissue for evaluation. } \\
\dagger \text { Specimens for which reticulin staining was performed ( } 30 \text { biopsies, } 12 \text { autopsies). } \\
\text { †Specimens for which Masson trichrome staining was performed. }\end{array}$} \\
\hline
\end{tabular}


Table 5. Univariate and Multivariate Logistic Regression Demonstrating Associations with Biopsy Findings

\begin{tabular}{|c|c|c|c|}
\hline Factor & OR $(95 \% \mathrm{CI})$ & $\begin{array}{c}\text { Univariate } P \\
\text { Value }\end{array}$ & $\begin{array}{c}\text { Multivariate } \\
P \text { Value }\end{array}$ \\
\hline \multicolumn{4}{|l|}{ Central venopathy } \\
\hline No. of abscess episodes & $9.35(1.04-83.3)$ & 0.033 & 0.045 \\
\hline No. of ALP elevations & $1.45(1.00-2.11)$ & 0.049 & NS \\
\hline \multicolumn{4}{|l|}{ Portal venopathy } \\
\hline Splenomegaly & $14.67(1.83-117.7)$ & 0.016 & 0.016 \\
\hline \multicolumn{4}{|l|}{ Acute cholangitis } \\
\hline No. of abscess episodes & $3.07(1.13-8.40)$ & 0.028 & 0.028 \\
\hline \multicolumn{4}{|l|}{ NRH } \\
\hline No. of ALP elevations & $1.20(1.00-1.45)$ & 0.045 & 0.045 \\
\hline
\end{tabular}



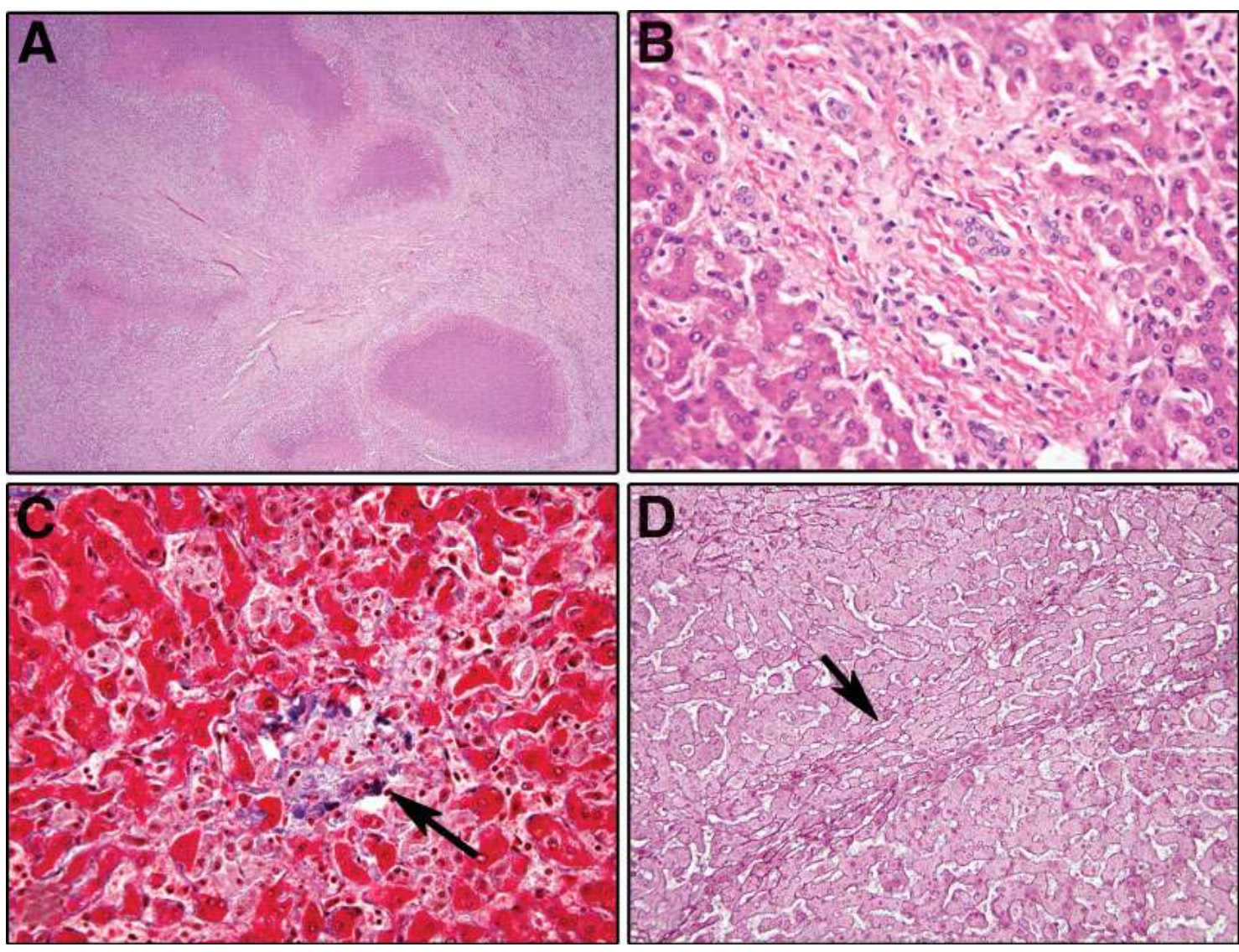

Figure 1. Hepatic pathology in CGD. (A) Multiloculated abscesses are present within dense fibroinflammatory connective tissue. The abscesses are irregular in outline and are surrounded by a thin cuff of epithelioid macrophages. The connective tissue around the abscesses contains remnants of portal areas, lymphocytes, eosinophils, and pigmented macrophages (hematoxylin-eosin stain). (Original magnification X40.) (B) Portal area showing obliteration of the normal portal vein. Small slit-like vessels may be seen along with the normal artery and vein. Only minimal inflammation is present, but pigmented macrophages are seen in the center of the portal area (hematoxylin-eosin stain). (Original magnification X400.) (C) A small central vein lumen is obliterated by loose connective tissue. Only the collagenous vein wall (arrow) remains as a marker of the vein's location. The surrounding sinuses are dilated and filled with pigmented macrophages (Masson trichrome stain). (Original magnification X400.) (D) Some of the specimens showed evidence of nodular regenerative hyperplasia. In this field, cords of narrowed, atrophic hepatocytes (arrow) are seen sandwiched between hypertrophic, widened liver cell plates (reticulin stain). (Original magnification X200.) 


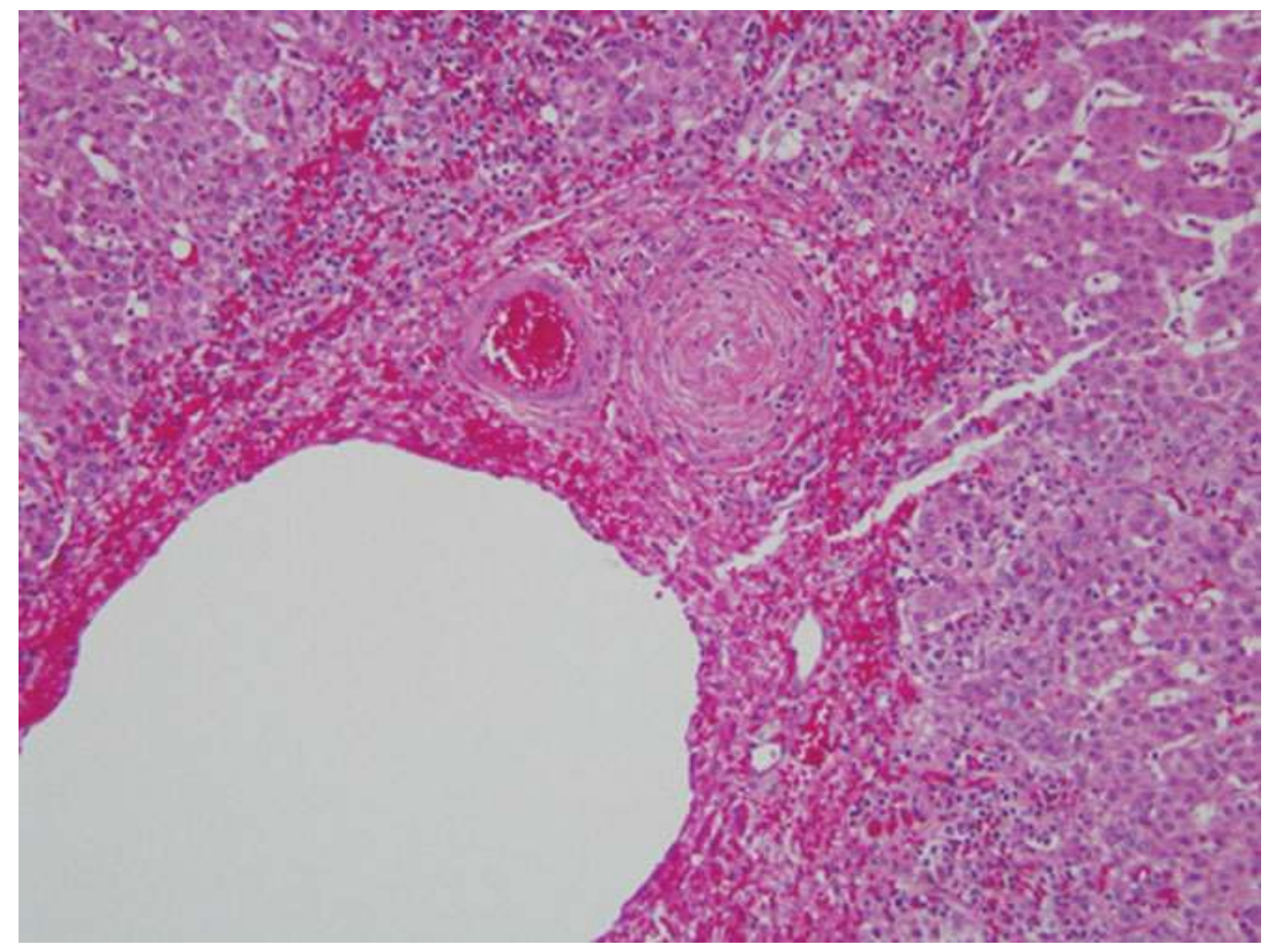

Figure 2. Obliterative duct lesion. A representative portal area from a hepatic resection specimen at a site distant from abscesses. The duct is obliterated and replaced by a concentric scar approximately the same size as the artery. The portal area also shows hemorrhage and mild peripheral chronic inflammation (hematoxylin-eosin stain; original magnification X100). 